\title{
Compra de alimentos da agricultura familiar pelo Programa Nacional de Alimentação Escolar (PNAE): estudo transversal com o universo de municípios brasileiros
}

\author{
Purchase of products directly from family farms \\ for the National School Feeding Program (PNAE): \\ cross-sectional study with the universe of brazilian municipalities
}

Patrícia Maria de Oliveira Machado ${ }^{1}$
Bethsáida de Abreu Soares Schmitz ${ }^{2}$
David Alejandro González-Chica ${ }^{3}$
Arlete Catarina Tittoni Corso ${ }^{1}$
Francisco de Assis Guedes de Vasconcelos ${ }^{1}$
Cristine Garcia Gabriel $^{1}$
${ }^{1}$ Centro Colaborador em Alimentação e Nutrição do Escolar do Estado de Santa Catarina, Universidade Federal de Santa Catarina. Campus Universitário, Centro de Ciências da Saúde, Departamento de Nutrição Sala 215, Trindade. 88040-900

Florianópolis SC Brasil. patriciamomachado@ gmail.com

${ }^{2}$ Departamento de Nutrição, Universidade de Brasília. Brasília DF Brasil.

${ }^{3}$ School of Medicine, NHMRC Centre of Research Excellence to Reduce Inequality in Heart Disease, The University of Adelaide. Adelaide SA Australia.
Abstract The integration of family farming with school meals has the potential to improve the variety of school menus thereby bringing the production and consumption of food into closer alignment. This study researched the Brazilian municipalities with respect to the purchase of food from family farms for the National School Food Program. It involved a cross-sectional study conducted via an electronic questionnaire sent to 5,565 municipalities in the country. The research included $93.2 \%$ of the municipalities ( $n=$ 5,184), 78.5\% of which acquired food from family farms, with the highest frequency (95.5\%) of the municipalities making the purchase in the southern region, and the lowest (67.9\%) in the Central West region. The large-scale municipalities, with mixed, decentralized or outsourced school food management, and without a nutritionist as technical manager, purchased food less frequently from family farms. The conclusion reached is that, despite the widespread acquisition of food from family farms in the program throughout the country, 50\% of municipalities did not invest the minimum required by law, requiring actions aimed at compliance with legislation and greater investment, especially in states and regions which revealed the greatest difficulties.

Key words School food, Family farming, Nutrition and food programs and policies
Resumo A integração entre a agricultura familiar e a alimentação escolar têm o potencial de melhorar a variedade dos cardápios escolares aproximando produção e consumo de alimentos. Este estudo caracterizou os municipios brasileiros quanto à compra de alimentos da agricultura familiar pelo Programa Nacional de Alimentação Escolar. Trata-se de estudo transversal realizado por meio de questionário eletrônico enviado aos 5.565 municípios do país. Participaram da pesquisa $93,2 \%$ dos municípios $(n=5.184)$. Destes, $78,5 \%$ adquiriram alimentos da agricultura $f a-$ miliar, destacando-se a região Sul, com a maior frequência de municípios realizando a compra (95,5\%), e a região Centro-Oeste com a menor (67,9\%). Os municípios de grande porte, com gestão da alimentação escolar do tipo mista, descentralizada ou terceirizada e sem nutricionista como responsável técnico, apresentaram menor frequência de compra de alimentos da agricultura familiar. Conclui-se que, apesar da ampla efetivação da aquisição de alimentos da agricultura familiar pelo programa em todo país, 50\% dos municípios não investiram o mínimo exigido em lei, demandando ações educativas e de assistência técnica direcionadas para o cumprimento da legislação, em especial nos estados e regiões que apresentaram maiores dificuldades.

Palavras-chave Alimentação escolar, Agricultura familiar, Programas e políticas de nutrição e alimentação 


\section{Introdução}

O Programa Nacional de Alimentação Escolar (PNAE) atendeu 41,5 milhões de estudantes da educação básica em todo o país, no ano de 2015, número que correspondeu a $20,3 \%$ da população brasileira $^{1}$. Sua capacidade de promover melhores hábitos alimentares e incentivar o comércio e a produção local de alimentos incluem este programa como estratégia fundamental de Segurança Alimentar e Nutricional (SAN) e efetivação do Direito Humano à Alimentação Adequada (DHAA), no Brasil².

Com a promulgação da Lei no $11.947 / 2009$ pelo Fundo Nacional de Desenvolvimento da Educação (FNDE), o PNAE modificou o escopo do processo de compra de alimentos para o programa, inserindo a obrigatoriedade dos municípios destinarem no mínimo 30\% dos recursos repassados para estados e municípios na compra direta de alimentos provenientes da agricultura familiar ${ }^{3}$, reafirmando o potencial para efetivação da $\mathrm{SAN}^{2}$. No ano de 2015, a capacidade de investimento na compra de alimentos da agricultura familiar pelo PNAE atingiu mais de 3,8 bilhões de reais, uma vez que o total de recursos repassados podem ser gastos nesta modalidade de aquisição, e um valor mínimo de 1,1 bilhão de reais, considerando apenas os $30 \%$ exigidos por lei ${ }^{1}$.

A parceria entre programas de alimentação escolar, a agricultura familiar e o incentivo à produção local de alimentos existe também em outros países como Estados Unidos ${ }^{4}$, Dinamar$\mathrm{ca}^{5}$, Reino Unido ${ }^{6}$, Itália ${ }^{7}$, Bolívia e Colômbia ${ }^{8}$, e alguns países da África ${ }^{9}$. Entretanto, o Brasil destaca-se pelo desenvolvimento de um programa de caráter universal, enquanto as estratégias internacionais correspondem a projetos localizados em determinadas escolas, municípios e regiões. Assim, estudos têm sido desenvolvidos no país para avaliar o potencial do PNAE como instrumento promotor de dinâmicas econômicas locais ${ }^{10,11}$.

Em um país de dimensões continentais como o Brasil, com o processo de descentralização administrativa e financeira em direção aos estados e municípios, de forma articulada à instância federal, por meio do órgão financiador e legislador do programa (o FNDE), estabelece-se a necessidade de análise continuada do cumprimento da legislação. Nesse quesito, destacam-se a Lei $n^{\circ}$ $11.947 / 2009^{3}$ e a Resolução no $26 / 2013^{12}$, no que se refere à compra direta de alimentos da agricultura familiar para a constante melhoria da execução do programa.
O objetivo deste estudo foi mapear e caracterizar a compra de alimentos da agricultura familiar pelo PNAE no universo de municípios brasileiros, segundo as regiões e estados, porte dos municípios, tipo de gestão do programa, presença do nutricionista como Responsável Técnico e valores de repasse do FNDE.

\section{Método}

\section{Tipo de estudo e amostra}

O estudo caracteriza-se como transversal, exploratório e descritivo. Os dados analisados fazem parte de projeto de pesquisa mais amplo desenvolvido pelo Centro Colaborador de Alimentação e Nutrição do Escolar do Estado de Santa Catarina (CECANE/SC). A amostra compreendeu todos os municípios brasileiros apontados pelo Instituto Brasileiro de Geografia e Estatística no ano de $2012(\mathrm{n}=5.565)^{13}$. Detalhes dos procedimentos metodológicos foram descritos em artigo publicado anteriormente ${ }^{14}$.

\section{Coleta de dados}

No período de outubro a dezembro de 2011 foi elaborada previamente uma lista de contatos de todos os municípios, com e-mail e telefones, por meio de consulta aos sites oficiais das prefeituras, além de acesso ao cadastro de responsáveis técnicos do PNAE no FNDE. Elaborou-se um questionário eletrônico por meio do aplicativo Google Docs ${ }^{\circledR}$ com perguntas pertinentes à temática abordada, direcionadas ao nutricionista responsável técnico do PNAE nos municípios ou aos secretários municipais de educação. $\mathrm{O}$ formulário online foi enviado às Secretarias Municipais de Educação entre os meses de fevereiro e outubro de 2012, com perguntas sobre a execução do programa no ano anterior. A equipe contou com nove colaboradores para a coleta de dados, entre bolsistas, estudantes, pós-graduandos e agentes do PNAE atuantes no CECANE/SC.

Para o monitoramento das respostas e correção do banco de dados gerado automaticamente, cada participante da coleta foi responsável por uma parcela de municípios, separados previamente de forma homogênea entre os estados. Foram realizadas três tentativas de contato via e-mail, sendo que o reenvio do questionário eletrônico aos municípios não respondentes aconteceu 15 dias após o primeiro envio. Ainda assim, aos municípios que permaneceram sem 
responder ao questionário, foram realizados até dois contatos telefônicos quinzenalmente com objetivo de esclarecer os objetivos da pesquisa e sensibilizar a participação. A coleta de dados foi realizada via telefone nos casos em que não houve possibilidade de obter as respostas via formulário online. Entre novembro de 2012 e fevereiro de 2013 foi realizada a limpeza e codificação do banco de dados.

Os dados referentes ao porte dos municípios foram coletados no sítio eletrônico IBGE Cida$d e{ }^{\circledR 13}$, e o valor de recursos repassados a cada município para a execução do programa no ano de 2011 foi disponibilizado em listagem completa pelo $\mathrm{FNDE}^{1}$.

\section{Variáveis independentes}

As variáveis independentes utilizadas neste estudo foram: a) Região do Brasil (Centro-Oeste, Nordeste, Norte, Sudeste, Sul); b) porte do município segundo o número de habitantes (< 20.000 , entre 20.001 a $100.000,>100.000$ ); c) tipo de gestão do PNAE no município (centralizada, descentralizada, terceirizada, mista) ${ }^{12}$ e d) presença do nutricionista como responsável técnico pelo PNAE (sim ou não).

\section{Variáveis dependentes}

As variáveis dependentes analisadas foram: a) realização da compra de alimentos da agricultura familiar (sim ou não); e b) percentual de recursos destinados à compra de alimentos da agricultura familiar em relação à verba total repassada pelo FNDE $(<30 \%$ de recursos e $\geq 30 \%$ recursos $)$.

\section{Análises}

Os dados foram processados em planilha no Microsoft Excel ${ }^{\circledR}$, e em seguida transferidos para o software Stata ${ }^{\circledR}$ 11.0. Foram realizadas análises conforme distribuição de frequências absolutas e relativas, acompanhadas do intervalo de confiança de 95\%. A estatística analítica, a fim de verificar a associação entre as variáveis dependentes e cada variável independente foi realizada pela análise bivariada por meio do teste qui-quadrado $\left(\chi^{2}\right)$ com correção de Yates $^{15}$. As análises tiveram nível de significância estatística considerando o valor de 5\% $(\mathrm{p}<0,05)$. Na distribuição espacial dos dados foi utilizado o software TabWin $32.0^{\circledR}$, disponibilizado pelo Ministério da Saúde para a construção do mapa com a malha municipal brasileira dividida em três categorias: não comprou da agricultura familiar; comprou da agricultura familiar no ano de 2011; município não participante da pesquisa.

\section{Aspectos éticos}

O projeto foi submetido e aprovado pelo Comitê de Ética em Pesquisa com Seres Humanos (CEPSH) da UFSC, de acordo com as normas estabelecidas pela Resolução 466/2012 do Conselho Nacional de Saúde.

\section{Resultados}

Este estudo contou com a participação de 93,2\% $(\mathrm{n}=5.184)$ do total de municípios brasileiros no ano de 2011 ( $\mathrm{n}=5.565)$. O comparativo das regiões brasileiras indicou maiores frequências na participação dos municípios da região Sul com $98,7 \%$ ( $\mathrm{p}<0,001)$, seguida das regiões Centro-Oeste $(97,6 \%)$ e Sudeste $(95,1 \%)$. Dentre os estados destaca-se a participação de todos os municípios do Mato Grosso do Sul, Espírito Santo e Santa Catarina (dados não apresentados em tabela).

Em relação ao número de habitantes dos municípios respondentes, percebeu-se a concentração de municípios de pequeno porte $(<20.000$ habitantes) com 70,3\% do total de respondentes (Tabela 1).

Com relação ao PNAE, a grande maioria dos municípios gerenciava o programa de forma centralizada $(92,0 \%)$ e possuía o nutricionista como responsável técnico $(94,3 \%)$. Sobre os valores de repasse do FNDE em 2011, notou-se a grande amplitude de valores recebidos pelos municípios respondentes ( $\mathrm{R} \$ 918,00-\mathrm{R} \$ 61.942 .260,00)$, com mediana de $\mathrm{R} \$ 114.420,00$ (Intervalo Interquartil $\mathrm{R} \$ 47.400,00$ - R\$283.560,00). Em relação aos valores destinados à agricultura familiar obteve-se a mediana de $\mathrm{R} \$ 27.276,40$ (Intervalo Interquartil R\$ 11.000,00 - R\$68.259,70) (Tabela 1).

Quanto à compra de produtos da agricultura familiar pelo PNAE, 78,5\% (IC95\% 77,4; 79,6) dos municípios brasileiros afirmaram realizar o processo de aquisição no ano de 2011. A frequência de realização da compra de produtos da agricultura familiar variou entre 67,9\% (IC95\% 63,6; 72,3 ) na região Centro-Oeste e 95,5\% (IC95\% 94,3 ; 96,6) na região Sul (dados não apresentados em tabela).

Na Figura 1 visualiza-se a representação espacial da compra da agricultura familiar pelos municípios, reiterando-se a região Sul como aquela 
Tabela 1. Caracterização dos municípios respondentes segundo região do Brasil, porte, tipo de gestão do Programa Nacional de Alimentação Escolar (PNAE), nutricionista como responsável técnico (RT) e valores de repasse do Fundo Nacional de Desenvolvimento da Educação (FNDE) ( $\mathrm{n}=5184)$. Brasil, 2011.

\begin{tabular}{|c|c|c|c|}
\hline & $\mathbf{N}$ & $\%$ & (IC95\%) \\
\hline \multicolumn{4}{|l|}{ Municípios participantes } \\
\hline Sim & 5184 & 93,2 & $(92,8 ; 93,2)$ \\
\hline Não & 381 & 6,8 & $(6,2 ; 7,5)$ \\
\hline \multicolumn{4}{|l|}{ Região do Brasil } \\
\hline Centro-Oeste & 455 & 8,8 & $(8,0 ; 9,5)$ \\
\hline Nordeste & 1581 & 30,5 & $(29,2 ; 31,8)$ \\
\hline Norte & 388 & 7,5 & $(6,8 ; 8,2)$ \\
\hline Sudeste & 1587 & 30,6 & $(29,6 ; 31,9)$ \\
\hline Sul & 1173 & 22,6 & $(21,5 ; 23,8)$ \\
\hline \multicolumn{4}{|l|}{ Porte do Município } \\
\hline Até 5.000 hab. & 1211 & 23,4 & $(22,2 ; 24,5)$ \\
\hline De 5001 a 20.000 & 2432 & 46,9 & $(45,5 ; 48,3)$ \\
\hline De 20.001 a 100.000 & 1269 & 24,5 & $(23,3 ; 25,6)$ \\
\hline De 100.001 a 500.000 & 235 & 4,5 & $(4,0 ; 5,1)$ \\
\hline Mais de $500.000^{\star}$ & 37 & 0,7 & $(0,5 ; 0,9)$ \\
\hline \multicolumn{4}{|l|}{ Tipo de gestão do PNAE } \\
\hline Centralizada & 4756 & 92,0 & $(91,3 ; 92,8)$ \\
\hline Descentralizada & 146 & 2,8 & $(2,4 ; 3,3)$ \\
\hline Terceirizada & 117 & 2,3 & $(1,8 ; 2,7)$ \\
\hline Mista & 149 & 2,9 & $(2,4 ; 3,3)$ \\
\hline \multicolumn{4}{|l|}{$\begin{array}{l}\text { Presença de nutricionista } \\
\text { (Responsável Técnico) }\end{array}$} \\
\hline $\operatorname{Sim}$ & 4883 & 94,3 & $(93,6 ; 95,0)$ \\
\hline Não & 298 & 5,7 & $(5,1 ; 6,4)$ \\
\hline \multicolumn{4}{|l|}{ Valor de repasse FNDE (2011) } \\
\hline $\begin{array}{l}1^{\circ} \text { quartil (R\$918,00 - } \\
49.080,00)\end{array}$ & 1311 & 25,5 & $(23,7 ; 25,8)$ \\
\hline $\begin{array}{l}2^{\circ} \text { quartil }(\mathrm{R} \$ 49.140,00- \\
117.000,00)\end{array}$ & 1284 & 25,0 & $(23,6 ; 25,9)$ \\
\hline $\begin{array}{l}3^{\circ} \text { quartil (R\$ } 117.180,00- \\
288.900,00)\end{array}$ & 1276 & 24,8 & $(24,0 ; 26,4)$ \\
\hline $\begin{array}{l}4^{\circ} \text { quartil }(\mathrm{R} \$ 289.020,00- \\
61.942 .260,00)\end{array}$ & 1267 & 24,7 & $(24,1 ; 26,4)$ \\
\hline
\end{tabular}

${ }^{*} \mathrm{IBGE}^{16}$.

Fonte: Dados da Pesquisa, 2011.

com maior frequência de compra, em contraposição as regiões Norte e Centro-Oeste, que apresentaram as menores frequências.

$\mathrm{Na}$ região Centro-Oeste, os menores percentuais da aquisição de produtos da agricultura familiar foram observados nos estados de Goiás e Distrito Federal, seguidos por Mato Grosso do Sul. Nos estados da região Nordeste, o Piauí possuía a menor frequência da realização da compra

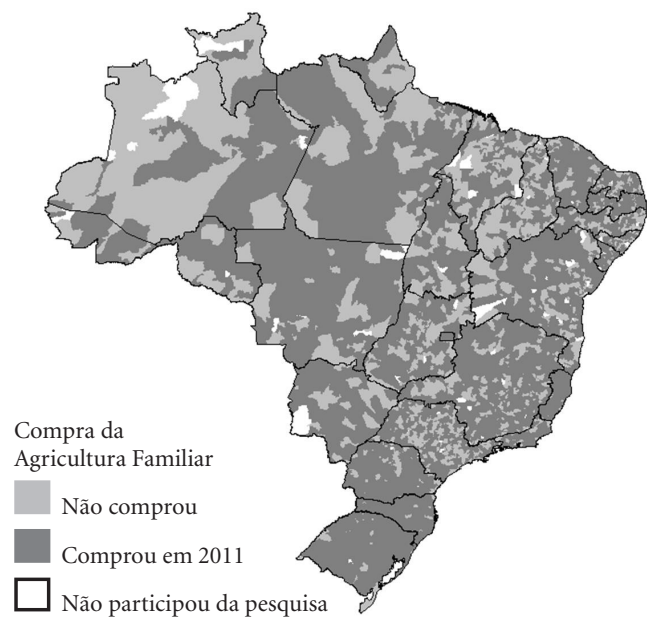

Figura 1. Mapa de distribuição espacial da compra da agricultura familiar pelo Programa Nacional de Alimentação Escolar (PNAE) nos municípios brasileiros. Brasil, 2011.

(55,0\%, IC95\% 47,7; 62,3), seguido por Alagoas com 60,7\% (IC95\% 50,3; 71,0). Destaca-se ainda o estado do Ceará com 91,8\% (IC95\% 87,6; $95,9)$ de realização da compra de produtos da agricultura familiar (dados não apresentados em tabela).

Na região Norte, os estados de Amapá e Roraima apresentaram as menores frequências de compra da agricultura familiar do Brasil, com $38,5 \%$ (IC95\% 7,9; 69,1) e 50,0 (IC95\% 16,8; 83,2 ). Espírito Santo destacou-se na região Sudeste como o estado com maior percentual de realização de compra (93,6\% IC95\% 88,0; 99,1), enquanto o estado de São Paulo possuía o conjunto de municípios com menor realização de compra da agricultura familiar $(66,2 \%$ IC95\% 62,4; 70,0). A região Sul apresentou maior homogeneidade na compra de alimentos da agricultura familiar com valores acima de $90 \%$ nos três estados, destacando-se o Rio Grande do Sul com 96,6\% de municípios que efetivavam a compra (IC95\% 95,0; 98,2) (dados não apresentados em tabela).

$\mathrm{Na}$ Tabela 2 observa-se que as menores frequências de compra de alimentos da agricultura familiar ocorreram em municípios de grande porte $(73,9 \%)$, enquanto que aqueles de pequeno e médio apresentaram frequências similares. Entre as regiões e estados notou-se a mesma tendência, 
Tabela 2. Associação entre a realização de compra de produtos provenientes da Agricultura Familiar para o Programa Nacional de Alimentação Escolar (PNAE) e o porte dos municípios, segundo as regiões e estados brasileiros. Brasil, 2011. (n=5141).

\begin{tabular}{|c|c|c|c|c|c|c|c|c|c|}
\hline & \multicolumn{3}{|c|}{$<20$ mil habitantes } & \multicolumn{3}{|c|}{ Entre 20 e 100 mil habitantes } & \multicolumn{3}{|c|}{$>100$ mil habitantes } \\
\hline & $\mathbf{N}$ & $\%(\text { IC 95\%) })^{\mathrm{a}}$ & $\mathbf{p}^{*}$ & $\mathbf{N}$ & $\%(\text { IC 95\%) })^{\mathrm{a}}$ & $\mathbf{p}^{*}$ & $\mathbf{N}$ & $\%(\text { IC 95\%) })^{\mathrm{a}}$ & $\mathbf{p}^{*}$ \\
\hline Brasil & 3606 & $79,4(78,0 ; 80,8)$ & $0,014^{\star *}$ & 1263 & $81,0(78,8 ; 83,3)$ & $0,001^{\star *}$ & 272 & $73,9(68,6 ; 79,2)$ & $0,002^{* *}$ \\
\hline Centro-Oeste & 342 & $63,1(57,9 ; 68,2)$ & 0,396 & 84 & $83,3(75,2 ; 91,5)$ & 0,003 & 20 & $84,8(67,5 ; 100,0)$ & 0,245 \\
\hline $\begin{array}{l}\text { Distrito Federal e } \\
\text { Goiás }\end{array}$ & 181 & $54,7(47,4 ; 62,0)$ & & 42 & $80,9(65,6 ; 93,9)$ & & 11 & $72,6(41,2 ; 100,0)$ & \\
\hline Mato Grosso & 109 & $76,1(68,0 ; 84,3)$ & & 21 & $90,5(76,8 ; 100,0)$ & & 6 & $100,0(-)$ & \\
\hline Mato Grosso do Sul & 52 & $65,4(52,0 ; 78,8)$ & & 21 & $81,0(62,3 ; 99,3)$ & & 3 & $100,0(-)$ & \\
\hline Nordeste & 1031 & $73,6(70,9 ; 76,3)$ & 0,396 & 477 & $77,1(73,3 ; 80,9)$ & 0,183 & 57 & $72,6(60,5 ; 84,7)$ & 0,357 \\
\hline Alagoas & 54 & $50,0(36,2 ; 63,8)$ & & 34 & $76,5(61,4 ; 91,5)$ & & 1 & $100,0(-)$ & \\
\hline Bahia & 226 & $78,3(72,9 ; 83,7)$ & & 143 & $81,8(75,4 ; 88,2)$ & & 16 & $87,5(69,3 ; 100,0)$ & \\
\hline Ceará & 89 & $92,1(86,4 ; 97,8)$ & & 75 & $92,0(85,7 ; 98,3)$ & & 6 & $83,3(40,5 ; 100,0)$ & \\
\hline Maranhão & 101 & $74,3(65,6 ; 82,9)$ & & 50 & $68,0(54,6 ; 81,4)$ & & 8 & $62,5(19,2 ; 100,0)$ & \\
\hline Paraíba & 167 & $88,6(83,7 ; 93,5)$ & & 29 & $89,6(77,9 ; 100,0)$ & & 5 & $100,0(-)$ & \\
\hline Pernambuco & 65 & $67,7(56,0 ; 79,4)$ & & 80 & $61,3(50,3 ; 72,2)$ & & 12 & $33,3(20,5 ; 64,6)$ & \\
\hline Piauí & 156 & $52,6(44,6 ; 60,5)$ & & 21 & $71,4(50,4 ; 92,5)$ & & 3 & $66,7(0,0 ; 100,0)$ & \\
\hline Rio Grande do Norte & 124 & $71,0(62,9 ; 79,1)$ & & 26 & $61,5(41,5 ; 81,6)$ & & 4 & $100,0(-)$ & \\
\hline Sergipe & 49 & $83,7(72,9 ; 94,4)$ & & 19 & $94,7(83,7 ; 100,0)$ & & 2 & $100,0(-)$ & \\
\hline Norte & 242 & $65,9(59,8 ; 71,9)$ & $<0,001$ & 124 & $82,0(75,2 ; 88,9)$ & 0,006 & 18 & $51,2(0,0 ; 100,0)$ & 0,321 \\
\hline Acre & 14 & $64,3(35,6 ; 93,0)$ & & 5 & $80,0(24,5 ; 100,0)$ & & 1 & $100,0(-)$ & \\
\hline Amapá & 9 & $22,2(11,7 ; 56,1)$ & & 2 & $100,0(-)$ & & 2 & $50,0(0,0 ; 100,0)$ & \\
\hline Amazonas & 20 & $55,0(31,1 ; 78,9)$ & & 23 & $86,9(72,1 ; 100,0)$ & & 2 & $100,0(-)$ & \\
\hline Pará & 38 & $55,3(38,7 ; 71,8)$ & & 72 & $76,4(66,3 ; 86,4)$ & & 8 & $87,5(57,9 ; 100,0)$ & \\
\hline Rondônia & 32 & $71,9(55,4 ; 88,3)$ & & 15 & $93,9(79,0 ; 100,0)$ & & 2 & $100,0(-)$ & \\
\hline Roraima & 10 & $40,0(3,1 ; 76,9)$ & & 1 & $100,0(-)$ & & 1 & $100,0(-)$ & \\
\hline Tocantins & 119 & $76,5(68,7 ; 84,2)$ & & 6 & $100,0(-)$ & & 2 & $100,0(-)$ & \\
\hline Sudeste & 1076 & $75,2(72,7 ; 77,8)$ & 0,060 & 370 & $78,9(74,7 ; 83,1)$ & 0,007 & 129 & $64,4(56,0 ; 72,7)$ & $<0,001$ \\
\hline Espírito Santo & 45 & $93,3(85,7 ; 100,0)$ & & 26 & $96,2(88,2 ; 100,0)$ & & 7 & $85,7(63,1 ; 96,8)$ & \\
\hline Minas Gerais & 629 & $78,7(75,5 ; 81,9)$ & & 151 & $87,4(82,1 ; 92,8)$ & & 25 & $80,0(63,1 ; 96,8)$ & \\
\hline Rio de Janeiro & 27 & $63,0(43,5 ; 82,4)$ & & 33 & $81,8(67,9 ; 95,7)$ & & 25 & $76,0(58,0 ; 94,0)$ & \\
\hline São Paulo & 375 & $68,3(63,5 ; 73,0)$ & & 160 & $67,5(60,2 ; 74,8)$ & & 72 & $52,8(41,0 ; 64,6)$ & \\
\hline Sul & 915 & $95,7(94,4 ; 97,0)$ & 0,584 & 208 & $94,2(91,0 ; 97,4)$ & 0,209 & 48 & $95,9(90,1 ; 100,0)$ & 0,521 \\
\hline Paraná & 301 & $93,7(90,0 ; 96,4)$ & & 65 & $92,3(85,6 ; 99,0)$ & & 17 & $100,0(-)$ & \\
\hline Rio Grande do Sul & 386 & $97,4(95,8 ; 99,0)$ & & 89 & $94,4(89,5 ; 99,3)$ & & 20 & $90,0(75,6 ; 100,0)$ & \\
\hline Santa Catarina & 228 & $95,6(92,9 ; 98,3)$ & & 54 & $96,3(91,1 ; 100,0)$ & & 11 & $100,0(-)$ & \\
\hline
\end{tabular}

a - Percentual obtido em relação aos municípios respondentes. ${ }^{*}$ Qui-quadrado de heterogeneidade. ${ }^{*}$ Valor $\mathrm{p}$ da diferença entre as regiões.

exceto para o Centro-Oeste com maior percentual de realização de compra para municípios de grande porte $(84,8 \%)$ quando comparados aos de pequeno porte $(63,1 \%)$ (Tabela 2$)$.

Nos estados da região Norte identificaram-se os menores percentuais de realização de compra da agricultura familiar nos municípios de pequeno porte ( $\mathrm{p}<0,001)$, principalmente no Amapá (22,2\%), seguido de Roraima (40,0\%), Amazonas e Pará (ambos 55,0\%). As regiões Sudeste e Sul apresentaram frequências mais homogêneas da realização da compra, independentemente do porte dos municípios. Na região Sudeste destacou-se mais uma vez o estado de São Paulo com menor percentual de realização de compra em duas categorias, em especial os municípios de grande porte com $52,8 \%$ (Tabela 2 ).

Na Tabela 3 observam-se maiores frequências de realização de compra da agricultura familiar entre os municípios brasileiros com gestão centralizada da alimentação escolar (80,3\%) e com a presença do nutricionista como responsável técnico $(80,7 \%)$, sendo que ambas as análises apresentaram $\mathrm{p}<0,001$. 
As menores frequências de realização de compra quando a gestão do PNAE era descentralizada, terceirizada ou mista foram observadas nas regiões Centro-Oeste $(63,6 \%)$ e Sudeste $(64,1 \%)$ e, nos estados de Pernambuco (36,4\%), Piauí (42,9\%), Goiás e Distrito Federal $(50,0 \%)$ e São Paulo (55,3\%) (Tabela 3). A análise da compra da agricultura familiar associada ao tipo de gestão terceirizada isoladamente foi realizada na região Sudeste, onde todos os estados possuíam municípios representantes deste tipo de gestão. Nesta região pode-se perceber que os municípios com terceirização da alimentação escolar realizavam a compra em menor proporção quando comparados aos outros tipos de gestão do programa, sendo a média da região de 51,6\% (IC95\% 39,4; 63,8) (dados não apresentados em tabela).

Quanto à presença do nutricionista como responsável técnico da alimentação escolar, as frequências mais baixas de compra foram observadas em estados sem este profissional, como o Rio de Janeiro (20,0\%), Goiás e Distrito Federal (28,6\%), Piaú e Maranhão (33,3\%) (Tabela 3). A região Sul apresentou o percentual de realização de compra acima da média de $90 \%$ nas demais associações, incluindo os estados em que a gestão não era centralizada. Entretanto, os municípios que não possuíam o nutricionista como responsável técnico apresentaram um percentual de compra de alimentos da agricultura familiar $22 \%$ menor do que os municípios que contavam com este profissional (Tabela 3 ).

Dentre os 3.817 municípios respondentes que realizavam a compra de produtos da agricultura familiar, 44,4\% (IC95\% 42,9; 46,0) aplicaram $30 \%$ ou mais dos recursos do FNDE. Alagoas e Rio Grande do Norte são os destaques da região Nordeste, com menores frequências de municípios adequados à legislação, com 25,5\% e 25,3\% dos municípios gastando $30 \%$ ou mais dos recursos, respectivamente $(\mathrm{p}<0,001)$ (Tabela 4$)$.

O Sudeste apresentava $41,2 \%$ dos municípios com gastos adequados à legislação, porém é a região que apresentava maior discrepância entre os estados que a compõem, sendo que nos dois extremos estavam Espírito Santo com 63,8\% de municípios aplicando mais que $30 \%$ dos recursos na compra da agricultura familiar, e de outro lado o estado de São Paulo com 29,6\%. Apesar da diferença entre os quatro estados da região apresentar-se maior que em outras regiões, $o$ valor $p$ encontrado foi considerado limítrofe para significância estatística $(\mathrm{p}=0,064)$ (Tabela 4).

A região Sul mais uma vez destacou-se entre as regiões do país com $67,0 \%$ dos municípios in-
Tabela 4. Distribuição dos municípios que aplicaram mais de $30 \%$ de recursos para a compra de produtos provenientes da Agricultura Familiar para o Programa Nacional de Alimentação Escolar (PNAE) em relação ao valor de repasse do Fundo Nacional de Desenvolvimento da Educação (FNDE), segundo as regiões e estados brasileiros. Brasil, 2011. $(n=3817)$.

\begin{tabular}{|c|c|c|c|}
\hline & \multirow[b]{2}{*}{$\mathbf{N}$} & \multicolumn{2}{|c|}{$\geq 30 \%$ dos recursos } \\
\hline & & $\%(\text { IC } 95 \%)^{a}$ & $p^{*}$ \\
\hline Brasil & 3817 & $44,4(42,9 ; 46,0)$ & $<0,001^{\star *}$ \\
\hline Região Centro-Oeste & 290 & $31,6(26,2 ; 37,0)$ & $<0,001$ \\
\hline Distrito Federal e Goiás & 138 & $31,2(23,3 ; 39,0)$ & \\
\hline Mato Grosso & 104 & $32,7(23,5 ; 41,9)$ & \\
\hline Mato Grosso do Sul & 49 & $30,6(17,2 ; 44,0)$ & \\
\hline Nordeste & 1100 & $32,0(29,2 ; 34,8)$ & $<0,001$ \\
\hline Alagoas & 51 & $25,5(13,1 ; 37,9)$ & \\
\hline Bahia & 284 & $32,0(26,6 ; 37,5)$ & \\
\hline Ceará & 152 & $36,8(29,1 ; 44,6)$ & \\
\hline Maranhão & 109 & $31,2(22,4 ; 40,0)$ & \\
\hline Paraíba & 165 & $38,2(30,7 ; 45,7)$ & \\
\hline Pernambuco & 91 & $26,4(17,1 ; 35,6)$ & \\
\hline Piauí & 91 & $29,7(20,1 ; 39,2)$ & \\
\hline Rio Grande do Norte & 99 & $25,3(16,5 ; 34,0)$ & \\
\hline Sergipe & 58 & $34,5(21,9 ; 47,1)$ & \\
\hline Norte & 258 & $26,4(21,5 ; 32,4)$ & $<0,001$ \\
\hline Acre & 14 & $21,4(0,0 ; 46,0)$ & \\
\hline Amapá & 5 & $20,0(0,0 ; 75,0)$ & \\
\hline Amazonas & 29 & $24,1(7,5 ; 40,5)$ & \\
\hline Pará & 80 & $25,0(15,3 ; 34,7)$ & \\
\hline Rondônia & 37 & $32,4(16,6 ; 48,2)$ & \\
\hline Roraima & 6 & - & \\
\hline Tocantins & 87 & $31,0(21,1 ; 40,9)$ & \\
\hline Sudeste & 1100 & $41,2(38,3 ; 44,1)$ & 0,064 \\
\hline Espírito Santo & 69 & $63,8(51,3 ; 75,4)$ & \\
\hline Minas Gerais & 600 & $47,0(43,0 ; 51,0)$ & \\
\hline Rio de Janeiro & 60 & $30,0(18,1 ; 41,9)$ & \\
\hline São Paulo & 371 & $29,6(25,0 ; 34,3)$ & \\
\hline Sul & 1068 & $67,0(64,1 ; 70,0)$ & $<0,001$ \\
\hline Paraná & 340 & $57,6(52,4 ; 62,9)$ & \\
\hline Rio Grande do Sul & 463 & $70,6(66,5 ; 74,8)$ & \\
\hline Santa Catarina & 265 & $72,8(67,4 ; 78,2)$ & \\
\hline
\end{tabular}

a - Percentual obtido em relação aos municípios respondentes. ${ }^{*}$ Quiquadrado de heterogeneidade. ${ }^{*}$ Valor $\mathrm{p}$ da diferença entre as regiões.

vestindo mais de $30 \%$ dos recursos na compra da agricultura familiar $(\mathrm{p}<0,001)$, sendo que o estado do Paraná apresentou a menor frequência $(57,6 \%)$ e Santa Catarina a maior $(72,8 \%)(\mathrm{Ta}-$ bela 4$)$. 


\section{Discussão}

Com base nos resultados encontrados pode-se perceber que a amostra investigada possui características que coincidem com a distribuição de municípios existentes nas cinco regiões do país e perfil dos municípios brasileiros, principalmente em relação à concentração de municípios de pequeno porte ${ }^{16}$. A amplitude do valor de repasse aos municípios para a execução do PNAE é justificada pelo fato deste valor ser calculado sobre o total de alunos matriculados ${ }^{17} \mathrm{e}$, desta forma, os municípios de grande porte recebem maior volume de verbas. Importante destacar que esta pesquisa não investigou os valores de contrapartida da gestão municipal, variável que pode ser importante em futuras pesquisas na associação com compra ou não da agricultura familiar e qualidade dos cardápios.

A aquisição de produtos da agricultura familiar sendo realizada por $78,5 \%$ dos municípios respondentes indica a ampla efetivação da medida proposta pelo FNDE em todo o território nacional. Em pesquisa realizada utilizando os dados do Demonstrativo Sintético Anual, enviado pelos municípios ao FNDE em relação ao ano de 2009, identificou-se que $47,4 \%$ dos municípios adquiriram alimentos da agricultura familiar ${ }^{18}$, ou seja, salvo diferenças metodológicas na coleta houve um indicativo de aumento de $31,1 \%$ ao longo de três anos.

No âmbito internacional, é importante ressaltar que não só o Brasil se preocupa em estabelecer relações de consumo sustentáveis, que direcionem recursos financeiros existentes para programas públicos de alimentação e nutrição visando o desenvolvimento da agricultura. Ao redor do mundo, diferentes programas de alimentação e nutrição destinados ao público escolar têm estabelecido processos de compra mais próximos do local de consumo e de pequenas produções, destacando-se internacionalmente a preferência por alimentos orgânicos. Tais ações além de visar o desenvolvimento da agricultura procuram, sobretudo, promover hábitos alimentares mais saudáveis, aproximando a cadeia de produção e de consumo ${ }^{4-8,10}$. No âmbito da América Latina cabe destacar que os programas de alimentação escolar se assemelham com o processo de evolução histórica do PNAE, uma vez que sofreram os mesmos mecanismos de ajuda internacional pela compra de alimentos excedentes da indústria alimentícia estadunidense. Segundo Belik e Souza ${ }^{8}$ apenas três países da América Latina desenvolveram programas em caráter universal, ou seja, de atendimento abrangente a todos os estudantes do país, são estes: Brasil, Chile e Panamá. Entretanto, mesmo sem possuir abrangência nacional, os programas de alimentação escolar da Bolívia e da Colômbia propõem o fortalecimento das pequenas produções agrícolas com a compra local de alimentos, assim como o Brasil.

Retornando-se aos resultados observados pela presente pesquisa, pode-se perceber que as maiores frequências da realização de compra foram encontradas entre os estados da região Sul $(95,5 \%)$, e as menores entre os estados do Centro-Oeste $(67,9 \%)$, dados semelhantes ao estudo de Saraiva et al. ${ }^{18}$, que avaliou as prestações de contas de 5.317 entidades executoras municipais do programa relativas ao ano de 2010. No estado de São Paulo, estudo com 645 municípios no ano de 2011 verificou que $50,0 \%$ destes já haviam adquirido alimentos da agricultura familiar ${ }^{19}$, valor menor ao encontrado nesta pesquisa $(66,2 \%)$.

Percebe-se que em regiões com características de produção agrícola, voltadas à agroindústria exportadora, ocorreram dificuldades dos agricultores familiares para atenderem ao volume necessário para a compra institucional do PNAE. Com base no Censo Agropecuário ${ }^{20}$ de 2006 a divisão entre os estabelecimentos da agricultura familiar segundo as regiões do Brasil se dá nas seguintes proporções: Nordeste (50\%), Sul (19\%), Sudeste $(16 \%)$, Norte (10\%) e Centro-Oeste (5\%), justificando a dificuldade de realização da compra na região Centro-Oeste, e em estados da região Norte como Amapá e Roraima, visto que estes ocupam o $4^{\circ}$ lugar na proporção de estabelecimentos rurais da agricultura familiar. Apesar da região Sul representar apenas 19\% dos estabelecimentos da agricultura familiar do país ${ }^{20}$, os valores de produção total de alimentos nesta região são bem maiores quando comparados com a região Nordeste $^{21}$, o que pode explicar os achados do presente estudo, que indicaram $95,5 \%$ de compra da agricultura familiar nos municípios do Sul.

Ainda assim, o potencial de qualificação dos hábitos, por meio do consumo de alimentos mais saudáveis, da diversificação do cardápio escolar ${ }^{22}$ e do acesso a alimentos orgânicos provenientes da compra direta da agricultura familiar são tratados na literatura em diversos locais, independentemente do número de estabelecimentos da agricultura familiar, como nas experiências municipais de Dois Irmãos (RS) ${ }^{23}$, Araripe $(\mathrm{CE})^{24}$, Espera Feliz $(\mathrm{MG})^{25}$ e em 82 municípios do estado de São Paulo ${ }^{26}$. Ainda, o incentivo à comercialização de alimentos e fortalecimento da agricultura familiar são tratados em outros estudos, 
a exemplo dos produtores de frutas do estado de São Paulo ${ }^{27}, 102$ municípios de territórios rurais do Rio Grande do Sul ${ }^{28}, 19$ cooperativas de sete mesorregiões do estado de Minas Gerais ${ }^{29}$, entre outros $^{30}$.

Nas análises de associação, o porte municipal mostrou-se determinante nos percentuais de realização da compra de produtos da agricultura familiar uma vez que os municípios de grande porte demonstraram menor capacidade de realização de compra (entre 10-20\% a menos), em especial nos estados de Amapá, Roraima e São Paulo. Em relatório organizado por Corá e Belik $^{31}$ sobre o processo de compras da agricultura familiar no estado de São Paulo, verificou-se que alguns municípios, principalmente os de maior porte, tiveram dificuldades na aquisição uma vez que os agricultores familiares não possuíam quantidades suficientes para o atendimento das necessidades do programa. Ainda assim, relatouse o caso de sucesso do município de São Bernardo do Campo que apesar de ser um município predominantemente industrial e de grande porte, investiu na articulação contínua com as organizações de produtores, não só entre os gestores, mas também entre merendeiras e agricultores, adequando o cardápio e a logística à realidade do município ${ }^{31}$.

No que se refere ao tipo de gestão do PNAE, a gestão centralizada foi predominante na grande maioria dos municípios, sendo que a compra da agricultura familiar se apresentou maior neste tipo de gestão. Para estabelecer a aquisição de produtos da agricultura familiar quando o responsável pela execução da compra era a escola, no caso da gestão descentralizada ou mista, ou ainda quando o município contava com empresas contratadas (terceirização), a compra da agricultura familiar foi entre $10-30 \%$ menor quando comparada à gestão centralizada. Estudos sobre o tipo de gestão do PNAE no país são escassos. Existe uma autonomia para as gestões municipais escolherem sua forma de gestão. No entanto, cabe aqui destacar que a região Sudeste mostrou maior frequência de adesão às terceirizações da alimentação escolar no país diante da amostra analisada, seguida da região Nordeste, e coincidentemente com valores menores de realização da compra da agricultura familiar pelos municípios. Ressalta-se ainda a inexistência de legislação específica para regular tal modelo, porém não se altera a necessidade de cumprir os $30 \%$ de compra direta da agricultura familiar ${ }^{5}$.

Neste estudo, a compra de alimentos da agricultura familiar mostrou-se relativamente maior nos municípios com a presença do nutricionista como responsável técnico inclusive na região Sul, identificando como potencial para compra da agricultura familiar o cumprimento da contratação deste profissional pelos municípios. Por exemplo, no estado de São Paulo a diferença entre os municípios com o nutricionista como responsável técnico incrementou em 31\% de efetivação da compra da agricultura familiar. Em vários estados a diferença no percentual de municípios que compram da agricultura familiar entre quem possui o nutricionista é de 2-4 vezes maior.

O FNDE estabelece o nutricionista como responsável técnico do $\mathrm{PNAE}^{32}$ e o Conselho Federal de Nutricionistas (CFN) atribui como responsabilidade deste profissional no programa, coordenar a execução incluindo a compra de alimentos ${ }^{33}$. Em outras análises a presença do nutricionista na alimentação escolar mostrou-se fundamental não apenas para a compra de alimentos, mas também para o incentivo a outros pontos da cadeia produção-consumo, uma vez que a realização de atividades educativas nas escolas para o uso e consumo de alimentos da agricultura familiar e orgânicos esteve intimamente relacionado à presença de nutricionistas, à carga horária de trabalho das mesmas e ao tempo de vínculo ${ }^{13}$.

Ressalta-se que apesar dos dados identificarem o nutricionista contratado na maior parte dos municípios, outros estudos ${ }^{34,35}$ identificaram que a adequação do número de profissionais em relação ao número de alunos ainda não é realiza$\mathrm{da}$, apontando para necessidade de constante capacitação e formação de profissionais de Nutrição alinhados ao desenvolvimento das políticas públicas de SAN como o PNAE e adequação do número de profissionais pelo total de estudantes.

Apesar do número de municípios que realizaram a compra da agricultura familiar, percebeuse que uma parte dos municípios ainda investia menos recursos do que o mínimo de $30 \%$ estabelecido em lei, com destaque para as regiões Norte e Centro-Oeste. Esta situação pode ser reflexo de uma série de fatores como a produção agrícola, falta de articulação entre gestores e agricultores, e dificuldades logísticas. Há a necessidade de reversão do desmantelamento da assistência técnica e extensão rural ocorrido na década de 1990, e em tentativa de ascensão desde 2003 no Brasil. Neste processo, destaca-se que o acesso do produtor familiar à assistência técnica no país é relativamente menor quando comparada a assistência técnica rural ofertada aos grandes produtores. Além disso, o crédito agrícola segue a lógica comercial 
e bancária de incentivos financeiros, não alinhados à necessidade de produção agrícola sustentável com vistas a soberania alimentar ${ }^{36,37}$.

Em relação ao valor de recursos utilizados para a compra de alimentos da agricultura familiar sugere-se que as futuras comparações utilizem o sistema de prestação de contas do FNDE que à época desta pesquisa não recolhia tais informações. Acredita-se que o incremento da compra da agricultura familiar pelo PNAE pode apoiar-se no incentivo à assessoria técnica ao desenvolvimento deste modo de produção por meio do Programa Nacional de Fortalecimento da Agricultura Familiar (PRONAF) e no apoio à alimentação escolar prestado pelos Centros Colaboradores em Alimentação e Nutrição do Escolar ligados ao $\mathrm{FNDE}^{38}$.

Os achados desta pesquisa apontam principalmente para a necessidade de expansão do número de nutricionistas e cumprimento das normativas por todos os executores do PNAE, em especial para aqueles municípios que apresentaram maiores dificuldades na aquisição de produtos da agricultura familiar sem a presença do profissional.

Como limitações deste estudo, cita-se a não inclusão das secretarias estaduais de educação na coleta de dados, e a possibilidade do não entendimento pleno de algumas perguntas por se tratar de uma pesquisa on-line. Para minimizar esta limitação, foram estabelecidos canais de comunicação com a equipe de coleta por meio de e-mail e contato telefônico. Em pesquisas de caráter universal, esta metodologia de coleta é bastante utilizada, permitindo o alcance da maioria dos municípios. Ainda, não foram obtidas informações sobre o número de nutricionistas existentes em cada município, fator que teria sido importante não apenas para avaliar o investimento de no mínimo 30\% dos recursos enviados pelo FNDE na compra de alimentos da agricultura familiar, mas também por ser uma variável que poderia interferir nos resultados das presentes análises.

\section{Considerações finais}

O presente artigo apresenta os dados de pesquisa realizada com mais de $90 \%$ dos municípios brasileiros. Destaca-se que estes foram pesquisados por meio de coleta de dados primários e secundários, com o rigor metodológico inerente a investigações deste porte. Assim, ressalta-se o ineditismo da pesquisa em questão, que pretende colaborar para o avanço das ações relacionadas à aquisição institucional de alimentos da agricultura familiar para o PNAE.

A medida legal proposta em 2009 permitiu a ampla efetivação da compra de alimentos provenientes da agricultura familiar pelo PNAE em todo o território nacional, modificando o escopo da compra de alimentos no programa. Entretanto, aponta-se a necessidade de ações direcionadas para o cumprimento da legislação (mínimo de $30 \%$ ) e ampliação dos investimentos, em especial nos estados e regiões que apresentaram maiores dificuldades, como no Centro-Oeste, região onde predomina a agricultura intensiva e de larga escala.

Verificou-se que os municípios que adotaram gestão da alimentação escolar do tipo mista, descentralizada ou terceirizada, atribuindo papel decisório da compra de alimentos às unidades escolares ou a empresas privadas, não realizaram a compra de alimentos provenientes da agricultura familiar com a mesma frequência que os municípios com gestão centralizada do programa, o que pode indicar a necessidade de maior controle, regulamentação e assistência para tais modalidades de gestão.

Ainda, os achados deste estudo atentam para a importância do nutricionista como responsável técnico pelo PNAE nos municípios, sobretudo para o incentivo à compra de alimentos da agricultura familiar.

Sugere-se a realização de mais pesquisas sobre o tema, que explorem as necessidades dos municípios em relação à assessoria técnica na área de gestão das políticas públicas e no desenvolvimento da agricultura familiar, além da articulação dos dados investigados a outras características municipais, como tempo de emancipação político-administrativa, índice de desenvolvimento humano e taxa de urbanização, identificando as relações de desenvolvimento das cidades e do acesso a alimentos provenientes da agricultura familiar.

Acrescenta-se ainda a necessidade de investigações que incorporem os elementos de produção de alimentos em volume por região e estado, a fim de estabelecer a relação entre oferta e demanda dos mercados institucionais, como a alimentação escolar, e assim diagnosticar as demandas locais para efetividade da soberania alimentar e promoção de hábitos alimentares saudáveis. 


\section{Colaboradores}

PMO Machado, BAS Schmitz, DA GonzálezChica e ACT Corso participaram da concepção da pesquisa, coleta e análise de dados. PMO Machado elaborou o manuscrito. BAS Schmitz, DA González-Chica, FAG Vasconcelos e CG Gabriel fizeram a revisão crítica do mesmo.

\section{Agradecimentos}

Projeto financiado pelo Fundo Nacional de Desenvolvimento da Educação. Termo de Cooperação UFSC-FNDE.

\section{Referências}

1. Brasil. Ministério da Educação. Fundo Nacional de Desenvolvimento da Educação - FNDE. Repasses financeiros. [acessado 2016 Set 25]. Disponível em: http://www. fnde.gov.br/programas/alimentacao-escolar/alimentacao-escolar-consultas/repasses-financeiros

2. Siqueira RL, Cotta RMM, Ribeiro RCL, Sperandio N, Priore SE. Análise da incorporação da perspectiva do direito humano à alimentação adequada no desenho institucional do programa nacional de alimentação escolar. Cien Saude Colet 2014; 19(1):301-310.

3. Brasil. Lei no 11.947 , de 16 de junho de 2009. Dispõe sobre o atendimento da alimentação escolar e do Programa Dinheiro Direto na Escola aos alunos da educação básica. Diário Oficial da União 2009; 17 jun.

4. O'toole TP, Anderson S, Miller C, Guthrie J. Nutrition services and foods and beverages available at school: results from the School Health Policies and Programs Study 2006. J Sch Health 2007; 77(1):500-521.

5. He C, Mikkelsen BE. Organic school meals in three Danish municipalities. Bioforsk Report 2009; (66):44.

6. Morgan K, Soninno R. Empowering consumers: the creative procurement of school meals in Italy and the UK. International Journal of Consumer Studies 2007; 31(1):19-25.

7. Nielsen T, Nölting B, Kristensen NH, Løes AK. A comparative study of the implementation of organic food in school meal systems in four European countries. Bioforsk Reports 2009; 4(145):34.

8. Belik W, Souza LR. Algumas Reflexões sobre os Programas de Alimentação Escolar na América Latina. Planejamento e políticas públicas 2009; 33(1):103-122.

9. Larsen AF, Lilleør HB. Beyond the Field: The Impact of Farmer Field Schools on Food Security and Poverty Alleviation. World Development 2014; 64(1):843-859.

10. Peixinho A, Balaban D, Rimkus L, Schwartzman F, Galante AP. A Alimentação Escolar no Brasil e nos Estados Unidos. O Mundo da Saúde 2011; 35(2):128-138.

11. Belik W, Chaim NA. O Programa Nacional de Alimentação Escolar e a gestão municipal: eficiência administrativa, controle social e desenvolvimento local. Rev. Nutr. 2009; 22(5):595-607.

12. Brasil. Ministério da Educação. Fundo Nacional de Desenvolvimento da Educação (FNDE). Resolução CD/ FNDE $n^{\circ} 26$ de 17 de junho de 2013. Dispõe sobre o atendimento da alimentação escolar aos alunos da educação básica no Programa Nacional de Alimentação Escolar - PNAE. Diário Oficial da União 2009; 18 jun.

13. Instituto Brasileiro de Geografia e Estatística (IBGE). Cidades@. [acessado 2016 Set 25]. Disponível em: http://www.cidades.ibge.gov.br/xtras/home.php

14. Vieira TV, Corso ACT, González-Chica DA. Organic food-related educational actions developed by dieticians in Brazilian municipal schools. Rev. Nutr. 2014; 27(5):525-535.

15. Barbetta PA. Estatística aplicada às ciências sociais. $7^{\mathrm{a}}$ ed. Florianópolis: EdUFSC; 2011.

16. Instituto Brasileiro de Geografia e Estatística (IBGE). Síntese de Indicadores Sociais: Uma análise das condições de vida da população brasileira. Rio de Janeiro: IBGE; 2010. [acessado 2016 Set 25]. Disponível em: http://www.ibge.gov.br/home/estatistica/populacao/ condicaodevida/indicadoresminimos/sinteseindicsociais2010/ Brasil. 
17. Ministério da Educação (MEC). Fundo Nacional de Desenvolvimento da Educação (FNDE). Resolução CD/FNDE no 38 de 16 de julho de 2009. Dispõe sobre o atendimento da alimentação escolar aos alunos da educação básica no Programa Nacional de Alimentação Escolar - PNAE. Diário Oficial da União 2009; 17 jul.

18. Saraiva EB, Silva APF, Sousa AA, Cerqueira GF, Chagas CMF, Toral N. Panorama da compra de alimentos da Agricultura familiar para o Programa Nacional de Alimentação Escolar. Cien Saude Colet 2013; 18(4):927935

19. Villar BS, Schwartzman F, Januario BL, Ramos JF. Situação dos municípios do estado de São Paulo com relação à compra direta de produtos da agricultura familiar para o Programa Nacional de Alimentação Escolar (PNAE). Rev. bras. epidemiol. 2013; 16(1):223-226.

20. Brasil. Instituto Brasileiro de Geografia e Estatística (IBGE). Censo Agropecuário, 2006. [acessado 2016 Set 25]. Disponível em: http://www.ibge.gov.br/home/ estatistica/economia/agropecuaria/censoagro/2006/ default.shtm.

21. Kamimura A, Oliveira A, Burani GF. A agricultura familiar no Brasil: um retrato do desequilíbrio regional. Interações 2010; 11(2):217-223.

22. Amorim ALB, Rosso VV, Bandoni DH. Acquisition of family farm foods for school meals: Analysis of public procurements within rural family farming published by the cities of São Paulo state. Rev. Nutr. 2016; 29(2):297-306.

23. Triches RM. Promoção do Consumo Alimentar Sustentável no Contexto da Alimentação Escolar. Trabalho, Educação e Saúde 2015; 13(3):757-771.

24. Marques AA, Fernandes MGM, Leite IN, Viana RT, Gonçalves MCR, Carvalho AT. Reflexões de agricultores familiares sobre a dinâmica de fornecimento de seus produtos para a alimentação escolar: o caso de Araripe, Ceará. Saude Soc 2014; 23(4):1329-1341.

25. Silva MG, Dias MM, Amorim Junior PCG. Mudanças Organizacionais em Empreendimentos de Agricultura Familiar a partir do Acesso ao Programa Nacional de Alimentação Escolar. Rev. Econ. Sociol. Rural 2015; 53(2):289-304.

26. Gonçalves HVB, Cunha DT, Stedefeldt E, Rosso VV. Family farming products on menus in school feeding: a partnership for promoting healthy eating. Ciência Rural 2015; 45(12):2267-2273

27. Souza-Esquerdo VF, Bergamasco SMPP. Análise sobre o acesso aos programas de políticas públicas da agricultura familiar nos municípios do circuito das frutas (SP). Rev. Econ. Sociol. Rural 2014; 52(Supl. 1):205-222.

28. Santos F, Fernandes PF, Rockett FC, Oliveira ABA. Avaliação da inserção de alimentos orgânicos provenientes da agricultura familiar na alimentação escolar, em municípios dos territórios rurais do Rio Grande do Sul, Brasil. Cien Saude Colet 2014; 19(5):1429-1436.
29. Costa BAL, Amorim Junior PCG, Silva MG. As Cooperativas de Agricultura Familiar e o Mercado de Compras Governamentais em Minas Gerais. Rev. Econ. Sociol. Rural 2015; 53(1):109-126.

30. Libermann AP, Bertolini GRF. Tendências de pesquisa em políticas públicas: uma avaliação do Programa Nacional de Alimentação Escolar - PNAE. Cien Saude Colet 2015; 20(11):3533-3546.

31. Corá MAJ, Belik W, organizadores. Projeto Nutre SP: análise da inclusão da agricultura familiar na alimentação escolar no estado de São Paulo. São Paulo: Instituto Via Pública, Ministério do Desenvolvimento Agrário; 2012.

32. Brasil. Ministério da Educação. Fundo Nacional de Desenvolvimento da Educação (FNDE). Resolução CD/FNDE no 32 de 10 de agosto de 2006. Estabelecer as normas para a execução do Programa Nacional de Alimentação Escolar - PNAE. Diário Oficial da União 2009; 11 ago.

33. Brasil. Conselho Federal de Nutricionistas. Resolução $n^{\circ} 465$ de 23 de agosto de 2010. Dispõe sobre as atribuições do Nutricionista, estabelece parâmetros mínimos de referência no âmbito do Programa de Alimentação Escolar (PAE) e dá outras providências. Diário Oficial da União 2010; 25 ago.

34. Chaves LG, Santana TCM, Gabriel CG, Vasconcelos FAG. Reflexões sobre a atuação do nutricionista no Programa Nacional de Alimentação Escolar no Brasil. Cien Saude Colet 2013; 18(4):917-926.

35. Gabriel CG, Calvo MCM, Ostermann RM, Vasconcelos FAG. Avaliação da gestão municipal do Programa Nacional de Alimentação Escolar nos maiores municípios de Santa Catarina. Cad Saude Publica 2014; 30(9):2017-2023.

36. Real LCV, Schneider S. O uso de programas públicos de alimentação na reaproximação do pequeno produtor com o consumidor: o caso do programa de alimentação escolar. Estudo \& Debate 2011; 18(Supl. 2):57-79.

37. Bonnal P, Maluf RS. Políticas de desenvolvimento territorial e multifuncionalidade da agricultura familiar no Brasil. Política \& Sociedade 2011; 8(Supl. 14):211-250.

38. Nogueira RM, Barone B, Barros TT, Guimarães KRLSLQ, Rodrigues NSS, Behrens JH. Sixty years of the National Food Program in Brazil. Rev. Nutr. 2016; 29(2):253-267.

Artigo apresentado em 18/07/2016

Aprovado em 26/01/2017

Versão final apresentada em 28/01/2017 